\title{
Synchronization of Spontaneous Contraction Activity in Smooth Muscle of Urinary Bladder
}

\author{
W.A. van Duyl, A.A.M. van der Hoeven, and J.V. de Bakker \\ Department of Biomedical Physics and Technology, Erasmus University, \\ Rotterdam, The Netherlands
}

\section{INTRODUCTION}

Strips of bladder smooth muscle exhibit spontaneous contraction activity of a rhythmic nature, with varying periodicities and waveforms. Frequency analysis has revealed that most of the time two main frequencies can be distinguished: one of about two per minute and the other about twice this frequency [Duyl, 1985]. Hak et al. [1988] found that the lower frequency component is inhibited by an anticholinergicum (oxyphenomiumbromide), whereas the higher frequency component is not affected by this drug. This observation suggests that these frequency components may have distinct origins.

We observed and analyzed two aspects of the spontaneous activity simultaneously on strips of bladder tissue of the pig: The contraction force across the strip and the local microdisplacements within the strip. Frequency analysis has been done on both the force signal and the motion signals. Furthermore, we looked for motion patterns of the spontaneous activity in the tissue.

\section{MATERIALS AND METHODS}

Strips of urinary bladder of the pig $(30 \times 15 \mathrm{~mm}$, rostrocaudal) were clamped lengthwise between two clamps, one of which was connected to an isometric force transducer (compliance $200 \mathrm{~g} / \mathrm{mm}$ ). The strips were stretched until an initial force of $\sim 10 \mathrm{gf}$ was reached. The strips were immersed in a metabolic solution oxygenated by bubbling with a $\mathrm{CO}_{2}$ gas mixture. The temperature of the bath was controlled by a thermostat. After an equilibratium period of about $1 \mathrm{hr}$ spontaneous activity of the strip has been recorded during about $2 \mathrm{hr}$. Microdisplacements in the strip were measured by means of nine fibers $(0.4 \mathrm{~mm}$ diameter) arranged in a square matrix of $3 \times 3$ (interdistance $5 \mathrm{~mm}$ ) and stuck through the strip. The tips of the strips were

Received for publication May 10, 1990; accepted May 23, 1990.

Address reprint requests to W.A. van Duyl, Department of Biophysics and Technology, Erasmus University Rotterdam, P.O. Box 1738, 3000 DR Rotterdam, The Netherlands. 
situated just at the surface of the strip. Each fiber tip followed the local motion of the tissue, presenting negligible mechanical load. The fiber tips emitted light sequentially by means of LEDs placed at the other end of each fiber. The light spot of the activated fiber tip was projected via a lens onto a position-sensitive photo detector, which yielded electrical currents according to the $(x, y)$-coordinates of the spot. With this technique, the $(x, y)$-position signals of each fiber were obtained sequentially at a sampling rate of 1 per second and with a resolution in displacement of $10 \mu \mathrm{m}$.

Both the isometric force signal and the fiber tip displacement signals were stored in a personal computer and analyzed afterwards. Preliminary observations have been done concerning the effect on spontaneous activity of stepwise elongation of the strip and of the addition of acetylcholine to the bath.

\section{OBSERVATIONS}

Some conclusions are drawn directly from mere observation of the displacement signals [Hoeven et al., 1989]. The displacement signal of each fiber tip showed a multitude of patterns that changed in the course of time but always had a more or less rhythmic nature. Often a waxing and waning amplitude with a dominant frequency was seen. The motions of some fiber tips were similar, in phase (synchronized) or in antiphase, whereas the motions of other fiber tips were different. Generally speaking, the various patterns seen in the force signal are also seen in the displacement signals. However, in a particular strip the local motions in the tissue during a certain episode may be rather dissimilar to the simultaneously recorded force across the strip. Sometimes relatively large motions were associated with small variations in the force and conversely. Large force signals were associated with a high degree of synchronization of the motions. Sometimes the local motions were verysmall; however, each fiber tip continuously showed some rhythmic motion.

To find out when tissue between two fiber tips is shortening (s) (contracting) or distending (d), the displacement signals of neighboring fiber tips have been subtracted. These s/d signals showed that sometimes contraction waves propagated through the tissue, with a propagation velocity of $\sim 1 \mathrm{~mm} / \mathrm{sec}$. Such a propagating contraction wave was accompanied with a plateau in the force signal.

\section{FREQUENCY ANALYSIS}

Running power spectra of the s/d signals and of the force signal were obtained by calculating the FFT transform of successive time intervals of $256 \mathrm{sec}$, shifted in steps of $64 \mathrm{sec}$ (overlap), during $2 \mathrm{hr}$ of observation. It appeared that most of the time the running power spectra of the s/d signals contained two frequency lobes: one at a mean frequency of $0.02-0.05 \mathrm{~Hz}\left(\mathrm{f}_{1}\right)$ and the other at a frequency about twice as high $\left(f_{h}\right)$, i.e., similar to what has been found for the force signal. The frequencies of the peaks and the power distribution between them were not constant but varied during the $2 \mathrm{hr}$ period of observation in a way that indicates the existence of two independent rhythmic phenomena. When the lower frequency was not present or had disappeared, the higher could still remain powerful. The addition of acetylcholine to the bath, which yielded a pulse-like increase of the force signal, appeared to affect mainly the low frequency component by increasing its frequency and decreasing the amplitude of that rhythmic motion. In contrast a stepwise elongation of the strip, which yielded a 
similar pulse in the force, appeared to affect mainly the higher frequency component in a similar way as acetylcholine affected the lower frequency component.

By using the running phase spectra of the s/d signals and the force signal in combination with the running power spectra, we were able to derive contraction patterns that were distributed in the tissue strip. It appeared that for each frequency band another contraction pattern could be distinguished composed of areas that contracted in phase with areas that distended. Hence for the two different frequency components different patterns are coexistent. The patterns with different frequencies had overlapping areas. The patterns were not stationary but varied in the $2 \mathrm{hr}$ period of observation.

\section{CONCLUSIONS}

The spontaneous contraction force measured across a strip is rhythmic and is generally composed of two frequency lobes: $f_{1}=0.02-0.05 \mathrm{~Hz}$ and $f_{h}=0.05-0.1$ $\mathrm{Hz}$. These contractions are not a result of homogeneously distributed contractions in the tissue but a result of contraction patterns in parts of the tissue with a certain degree of synchronization. The high frequency motion pattern can be distinguished from the low frequency motion pattern. Each pattern changes by migrating and by decreasing or increasing the areas of synchronized rhythmic contractions. The order of magnitude of the propagation velocity of the contraction waves is $1 \mathrm{~mm} / \mathrm{sec}$. More evidence for our conclusion that the two rhythms are generated by different physiologic mechanisms is furnished by the observation that acetylcholine mainly affects the lower frequency component and that distension mainly affects the higher frequency component.

\section{DISCUSSION}

The presence and functional significance of spontaneous activity in the normal human urinary bladder has been a controversial subject for a long time [Levin et al., 1986; Potjer and Constantinou, 1989]. In pathological situations, spontaneous activity is related to bladder instability and usually is ascribed to a neurological disorder. On the other hand, spontaneous activity is commonly observed in bladder strip preparations of different species of animals [Sibley, 1985]. It is evident that spontaneous activity of bladder strips does not have a neurological origin but is more likely to be myogenic. Many authors have supposed that in the in vitro situation the spontaneous activity is an artefact [Levin et al., 1986]. Plum [1960] noticed that the spontaneous contractions in human bladder did not occur until a relatively high volume was obtained and hypothesized that the onset of the micturition reflex was triggered by these spontaneous contractions via afferent stimuli. Hence his concept is that the amount of spontaneous contractions has a physiological meaning and its generation is related to passing a threshold in tissue distension. With regard to the question of the existence of spontaneous contractions in the normal human bladder, we think it is relevant to remark that as far as we know no one has tried to find micromotion at such a small scale as we did in vitro, in which situation we found tissue never at rest and furthermore that local motions do not necessarily result in detectable variations in the overall force. 
The magnitude of tension waves appeared to be associated with the degree of synchronization of regional contractions. We will investigate whether tissue distension and degree of synchronization are correlated. In our opinion, the question about the role of spontaneous activity seen in vitro in the generation of regional spontaneous bladder contraction in situ, in particular as a factor in a neurological feedback loop, has not yet been definitely answered. Furthermore, in connection to our observation of the two different rhythmic phenomena, we refer to two particular types of bladder instabilities that are described in literature [Brading et al., 1986]. One type of instability is seen as a consequence of urethral obstruction, and the other type we refer to is described as idiopathic. It has been reported that bladder tissue of unstable obstructed bladders has an increased sensitivity to acetylcholine and has a changed innervation. On the other hand, in tissue of idiopathic unstable bladders, no such change in innervation has been found, although its sensitivity to low frequent electrical stimulation appeared to be higher than normal. The existence of two separate rhythmic spontaneous contractions and their selective sensitivity to acetylcholine and to distension suggests that the two mechanisms generating these activities may be the genesis of the two types of bladder instability mentioned above. We conclude that more investigation of spontaneous activity both in vivo and in vitro is needed to elucidate the nature and origin of both types of spontaneous contraction and how it is related to bladder instability.

\section{REFERENCES}

Brading AF, Mostwin JL, Sibley GNA, Speakman MJ: The role of smooth muscle and its possible involvement in diseases of the lower urinary tract. Clin Sci 70[Suppl. 14]:7-13, 1986.

Duyl WA van: Spontaneous contractions in urinary bladder smooth muscle: Preliminary results. Neurourol Urodynam 4:301-307, 1985.

Hak J, Griffiths DJ, Mastrigt R van, Koopal B, Kluck P: Quantitative study in vitro of the spontaneous contractions of the pig detrusor muscle and their frequency-selective inhibition by drugs. Neurourol Urodynam 7:471-481, 1988.

Hoeven AAM van der, Duyl WA van, Bakker JV de: Detection of spontaneous motion patterns in urinary bladder tissue in vitro. Proc. Int. Symp. Dysfunction of the Lower Urinary Tract, Nijmegen, March 3-4, 1989.

Levin RB, Ruggerie MR, Velagapudi S, Gordon D, Altman B, Wein AJ: Relevance of spontaneous activity to urinary bladder function: An in vitro and an in vivo study. J Urol 136:514-521, 1986.

Plum F: Autonomous urinary bladder activity in normal man. Neurol 2:497-503, 1960.

Potjer RM, Constantinou CE: Frequency of spontaneous contractions in longitudinal and transverse bladder strips. Am J Physiol 257:R781-R787, 1989.

Sibley GNA: A comparison of spontaneous and nerve-mediated activity in bladder muscle from man, pig and rabbit. J Physiol 354:431-443, 1984. 